\title{
D-Optimal Slope Design for Second Degree Kronecker Model Mixture Experiment With Three Ingredients
}

\author{
Ngigi Peter Kung'u ${ }^{1}$, J. K. Arap Koske ${ }^{2}$, Josphat K. Kinyanjui ${ }^{1}$ \\ ${ }^{1}$ Department of mathematics, statistics and actuarial science, Karatina University, Karatina, Kenya \\ ${ }^{2}$ Department of mathematics \& computer science, Moi University, Eldoret, Kenya
}

Correspondence: Thomas C. Keane, Director, ISES Labs, Department of Chemistry and Biochemistry, Russell Sage College, Troy, NY 12180, USA. E-mail: keanet@sage.edu

Received: October 24, 2019 Accepted: February 5, 2020 Online Published: February 28, 2020

doi:10.5539/ijsp.v9n2p30

URL: https://doi.org/10.5539/ijsp.v9n2p30

\begin{abstract}
This study presents an investigation of an optimal slope design in the second degree Kronecker model for mixture experiments in three dimensions. The study is restricted to weighted centroid designs, with the second degree Kronecker model. A well-defined coefficient matrix is used to select a maximal parameter subsystem for the model since its full parameter space is inestimable. The information matrix of the design is obtained using a linear function of the moment matrices for the centroids and directly linked to the slope matrix. The discussion is based on Kronecker product algebra which clearly reflects the symmetries of the simplex experimental region. Eventually the matrix means are used in determining optimal values of the efficient developed design.
\end{abstract}

Keywords: information matrix, moment matrix, optimal design, response surface methodology, weighted centroid design, Kiefer ordering

\section{Introduction}

This study deals with the exploration and optimization of response surface. This is a problem faced by experimenters in many technical fields, where in general the response of interest is affected by a set of independent factors. In this response surface methodology (RSM) problem we assume a response of interest is influenced by three factors with the intent of optimizing this response. The response in linked to the factors through a second degree polynomial model.

In this mixture experiment the response is a function of the proportions of each ingredient. Let $x_{i}$ represent the proportion of the $i t h$ ingredient in the mixture. Then, we have two conditions, $x_{i} \geq 0, i=1,2,3$ and $\sum_{i=1}^{3} x_{i}=1$. Evidently the levels of the factors $x_{i}$ are interdependent. The experimental region for the mixture problem is a two dimensional simplex.

\section{Materials and Methods}

Let $1_{\mathrm{m}}=(1, \ldots, 1)^{\prime} \in \mathfrak{R}^{m}$ be a unity vector. The experimental conditions $\mathrm{t}=\left(\mathrm{t}_{1}, \mathrm{t}_{2}, \ldots, \mathrm{t}_{\mathrm{m}}\right)$ with $\mathrm{t}_{\mathrm{i}} \geq 0$ of a mixture experiment are points in the probability simplex,

$$
T_{m}=\left\{t=\left(t_{1}, \quad t_{2}, \quad \ldots, \quad t_{m}\right)^{\prime} \in[0,1]^{m}: 1_{m}^{\prime} t=1\right\} .
$$

Under experimental conditions, $t \in T_{m}$, the response $Y_{t}$ is taken to be a quantitative random variable. The responses are assumed to be uncorrelated with equal but unknown finite variance say $\sigma^{2} \in(0, \infty)$. The design in point has finite number of support points.

This study adopts a second degree polynomial regression function with the expected response:

$$
E\left(Y_{t}\right)=f(t)^{\prime} \theta=\sum_{i=1}^{m} \theta_{i i} t_{i}^{2}+\sum_{\substack{i, j=1 \\ i<j}}^{m}\left(\theta_{i j}+\theta_{j i}\right) t_{i} t_{j}
$$


where $Y_{t}$, is the response under experimental condition $t_{t \in T_{m}}$, and $\theta=\left(\theta_{11}, \theta_{12}, \ldots, \theta_{m m}\right) \in \mathfrak{R}^{m^{2}}$ an unknown parameter. (see (Draper \& Pukelsheim, 1998)).

A general review of design environment is done by (Pukelsheim, 1993) while (Klein, 2004) showed that the class of weighted centroid designs with at least two ingredients is essentially complete for the Kiefer ordering, (Draper, Heilijers, $\&$ Pukelsheim, 2000). As a consequence, we restrict the study to weighted centroid design.

\section{General Design Problem}

The problem of finding a design with maximum information on the parameter subsystem $K^{\prime} \theta$ can be formulated as;

$$
\begin{aligned}
& \text { Maximize } \varphi_{p}\left(C_{k}(M(\tau))\right) \text { with } \tau \in T \\
& \text { Subject to } C_{k}(M(\tau)) \in P D(s) \tau \in T
\end{aligned}
$$

where $\mathrm{T}$ denotes the set of all designs $\mathrm{T}_{\mathrm{m}}$. The side condition $C_{k}(M(\tau)) \in P D(s)$ is equal to the existence of an unbiased linear estimator for $K^{\prime} \theta$ under $\tau$, Pukelsheim (1993). In which case, the design $\tau$ is called feasible for $K^{\prime} \theta$. Any design solving problem (2) above for a fixed $\mathrm{p} \in(-\infty, 1]$ is called $\phi_{p}-$ optimalfor $K^{\prime} \theta$ in $\mathrm{T}$. For all $\mathrm{p} \in(-\infty, 1]$, the existence of $\phi_{p}-$ optimaldesign for $K^{\prime} \theta$ is certain, (Pukelsheim, 1993).

\section{Moment Matrix}

An experimental design $\tau$ is a probability measure on the experimental domain with a finite number of support points. Each support point $\mathrm{s} \in \operatorname{supp}(\tau)$ directs the experimenter to take a proportion $\mathrm{T}(\{\mathrm{t}\})$ of all observations under experimental condition $\mathrm{T}$. The statistical properties of a design are reflected by its moment matrix:

$$
M(\tau)=\int_{\tau} f(t) f(t)^{\prime} d \tau \in N N D\left(m^{2}\right)
$$

where, $\mathrm{NND}\left(\mathrm{m}^{2}\right)$ denotes the cone of nonnegative definite $\mathrm{m}^{2} \times \mathrm{m}^{2}$ matrices. The entries of $\mathrm{M}(\tau)$ are fourth moments of $\tau$, since the regression function $f(t)$ is purely quadratic.

\section{Information matrix}

We use unit vectors $\mathrm{e}_{1}, \mathrm{e}_{2}, \mathrm{e}_{3}$ and set $\mathrm{e}_{\mathrm{ij}}=\mathrm{e}_{\mathrm{i}} \otimes \mathrm{e}_{\mathrm{j}}$ for $\mathrm{i}<\mathrm{j} \quad \mathrm{i}, \mathrm{j}=\{1,2,3\}$ and define the coefficient matrix

where

$$
K=\left(K_{1} ; K_{2}\right) \in \mathfrak{R}^{m^{2} \times\left(\begin{array}{c}
m+1 \\
2
\end{array}\right)}
$$

$$
\begin{gathered}
K_{1}=\sum_{i=1}^{m} e_{i i} e_{i}^{\prime} \\
\text { and } K_{2}=\frac{1}{m} \sum_{\substack{i, j=1 \\
i<j}}^{m}\left(e_{i j}+e_{j i}\right) E_{i j}^{\prime}
\end{gathered}
$$

Obtainable as follows:

From $e_{1}=\left(\begin{array}{lll}1 & 0 & 0\end{array}\right)^{\prime}, e_{2}=\left(\begin{array}{lll}0 & 1 & 0\end{array}\right)^{\prime}$ and $e_{3}=\left(\begin{array}{llll}0 & 0 & 1\end{array}\right)^{\prime}$ we have:

$$
\begin{aligned}
& e_{11}=e_{1} \otimes e_{1}=\left(\begin{array}{lllllllll}
1 & 0 & 0 & 0 & 0 & 0 & 0 & 0 & 0
\end{array}\right)^{\prime}, e_{22}=e_{1} \otimes e_{1}=\left(\begin{array}{lllllllll}
0 & 0 & 0 & 0 & 1 & 0 & 0 & 0 & 0
\end{array}\right)^{\prime}, \\
& e_{33}=e_{3} \otimes e_{3}=\left(\begin{array}{lllllllll}
0 & 0 & 0 & 0 & 0 & 0 & 0 & 0 & 1
\end{array}\right)^{\prime}, e_{12}=e_{1} \otimes e_{2}=\left(\begin{array}{lllllllll}
0 & 1 & 0 & 0 & 0 & 0 & 0 & 0 & 0
\end{array}\right)^{\prime}, \\
& e_{21}=e_{2} \otimes e_{1}=\left(\begin{array}{lllllllll}
0 & 0 & 0 & 1 & 0 & 0 & 0 & 0 & 0
\end{array}\right)^{\prime}, e_{13}=e_{1} \otimes e_{3}=\left(\begin{array}{llllllllll}
0 & 0 & 1 & 0 & 0 & 0 & 0 & 0 & 0
\end{array}\right)^{\prime}, \\
& e_{31}=e_{3} \otimes e_{1}=\left(\begin{array}{lllllllll}
0 & 0 & 0 & 0 & 0 & 0 & 1 & 0 & 0
\end{array}\right)^{\prime}, e_{23}=e_{2} \otimes e_{3}=\left(\begin{array}{lllllllll}
0 & 0 & 0 & 0 & 0 & 1 & 0 & 0 & 0
\end{array}\right)^{\prime},
\end{aligned}
$$




$$
\begin{gathered}
e_{32}=e_{3} \otimes e_{2}=\left(\begin{array}{lllllllll}
0 & 0 & 0 & 0 & 0 & 0 & 0 & 1 & 0
\end{array}\right)^{\prime}, E_{12}=\left(\begin{array}{lll}
1 & 0 & 0
\end{array}\right)^{\prime}, E_{13}=\left(\begin{array}{lll}
0 & 1 & 0
\end{array}\right)^{\prime} \text { and } \\
E_{23}=\left(\begin{array}{lll}
0 & 0 & 1
\end{array}\right)^{\prime}
\end{gathered}
$$

Therefore, we obtain;

$$
K_{1}=e_{11} e_{1}{ }^{\prime}+e_{22} e_{2}{ }^{\prime}+e_{33} e_{3}^{\prime}=\left(\begin{array}{ccc}
1 & 0 & 0 \\
0 & 0 & 0 \\
0 & 0 & 0 \\
0 & 0 & 0 \\
0 & 1 & 0 \\
0 & 0 & 0 \\
0 & 0 & 0 \\
0 & 0 & 0 \\
0 & 0 & 1
\end{array}\right)
$$

and

$$
K_{2}=\left(e_{12}+e_{21}\right) E_{12}^{\prime}+\left(e_{13}+e_{31}\right) E_{13}^{\prime}+\left(e_{23}+e_{32}\right) E_{23}^{\prime}=\left(\begin{array}{ccc}
0 & 0 & 0 \\
\frac{1}{3} & 0 & 0 \\
0 & \frac{1}{3} & 0 \\
\frac{1}{3} & 0 & 0 \\
0 & 0 & 0 \\
0 & 0 & \frac{1}{3} \\
0 & \frac{1}{3} & 0 \\
0 & 0 & \frac{1}{3} \\
0 & 0 & 0
\end{array}\right)
$$

Thus

$$
K=\left(\begin{array}{ll}
K_{1} & K_{2}
\end{array}\right)=\left(\begin{array}{cccccc}
1 & 0 & 0 & 0 & 0 & 0 \\
0 & 0 & 0 & \frac{1}{3} & 0 & 0 \\
0 & 0 & 0 & 0 & \frac{1}{3} & 0 \\
0 & 0 & 0 & \frac{1}{3} & 0 & 0 \\
0 & 1 & 0 & 0 & 0 & 0 \\
0 & 0 & 0 & 0 & 0 & \frac{1}{3} \\
0 & 0 & 0 & 0 & \frac{1}{3} & 0 \\
0 & 0 & 0 & 0 & 0 & \frac{1}{3} \\
0 & 0 & 1 & 0 & 0 & 0
\end{array}\right)
$$

The full parameter vector $\theta \in \mathfrak{R}^{m^{2}}$ for model equation (1) is not estimable. We select a maximal sub parameter vector:

$$
K^{\prime} \theta=\left\{\begin{array}{l}
\left(\theta_{i i}\right)_{1 \leq i \leq m} \\
\frac{1}{m}\left(\theta_{i j}+\theta_{j i}\right)_{1 \leq i<j \leq m}
\end{array}\right\} \in \mathfrak{R}^{\left(\begin{array}{c}
m+1 \\
2
\end{array}\right)}
$$

for all

$$
\theta \in \mathfrak{R}^{m^{2}}
$$

To optimize the response, we focus on the movement of the design center along the direction of the directional derivatives of the response function, that is, $\frac{\partial Y_{t}}{\partial t}$. Since the designs that attain certain properties in $\mathrm{Y}$ (estimated 
response) do not enjoy the same properties for the estimated derivatives (slopes), we consider experimental designs that are constructed with derivatives in mind, (Murty \& Studden, 1972) and (Ott \& Mendenhall, 1972).

In practice, it is often of interest to investigate the slope of the response surface at a point $t$, not only over the axial directions, but also over any specified direction. We develop the concept of robust slope over all directions. Define D, a matrix arising from the differentiation of $f(t)^{\prime} \theta$ with respect to each of the $\mathrm{m}$ independent factors, (see (Sung, Hyang, \& Rabindra, 2009)). That is;

$$
D=\left(\frac{\partial f^{\prime}(t)}{\partial t_{1}}, \frac{\partial f^{\prime}(t)}{\partial t_{2}}, \ldots, \frac{\partial f^{\prime}(t)}{\partial t_{m}}\right)^{\prime}, \quad \text { where, } f(t)=t \otimes t
$$

An important matrix for the design with three ingredients is the adjusted $3 \times 6$ slope matrix $H_{0}=D K$.

The amount of information a design contains on $\mathrm{K}^{\prime} \theta$ is captured by the information matrix:

$$
\mathrm{C}_{\mathrm{k}}(\mathrm{M}(\tau))=\min \left\{\operatorname{LM}(\tau) \mathrm{L}, \quad \mathrm{L} \in \mathfrak{R}^{\left(\begin{array}{c}
m+1 \\
2
\end{array}\right) \times m^{2}} ; \mathrm{LK}={ }^{I}\left(\begin{array}{c}
m+1 \\
2
\end{array}\right)\right\}
$$

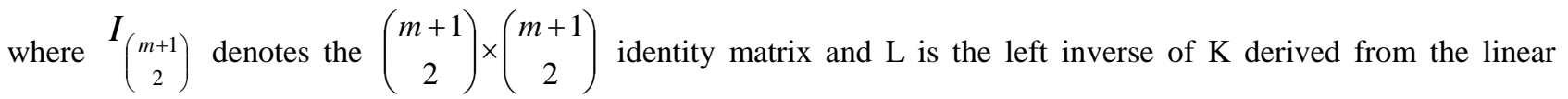
relation, $L=\left(K^{\prime} K\right)^{-1} K^{\prime}$. The information matrices for $\mathrm{K}^{\prime} \theta$ takes the form:

$$
C_{0}=L M(\tau) L^{\prime} \in N N D\left(\left(\begin{array}{c}
m+1 \\
2
\end{array}\right)\right)
$$

Thus the information matrices for $\mathrm{K}^{\prime} \theta$ are linear transformations of the moment matrices.

We then consider optimizing the information matrices for $\mathrm{K}^{\prime} \theta$ of the form:

$$
C=H_{0} C_{0} H_{0}^{\prime} \in N N N D(m)
$$

\section{Optimality Criteria}

We will compute optimal design for the polynomial fit model using matrix mean $\phi_{p}$ which is an information function (Pukelsheim, 1993). For an information matrix $\mathrm{C}_{\mathrm{k}}(\mathrm{M}(\tau)) \in \mathrm{PD}(\mathrm{m})$ the kiefers $\phi_{p}$-criteria are defined by:

$$
\phi_{p}(C)=\left\{\begin{array}{cc}
\lambda_{\min }(C) \text { if } & p=-\infty \\
\operatorname{det}(C)^{\frac{1}{\left(\begin{array}{c}
(+1 \\
2
\end{array}\right)}} \text { if } & p=0 \\
{\left[\begin{array}{c}
\frac{1}{\left(\begin{array}{c}
m+1 \\
2
\end{array}\right)} \text { trace }^{p} \\
{ }^{p}
\end{array}\right]^{p}} & p \in[-\infty ; 1] \backslash\{0\}
\end{array}\right.
$$

where $\lambda_{\min }(\mathrm{C})$ refers to the smallest eigenvalue of $\mathrm{C}$. By definition $\phi_{p}(\mathrm{C})$ is a scalar measure which is a function of the eigenvalues of $\mathrm{C}$ for all $\mathrm{p} \in[-\infty ; 1]$. (Pukelsheim, 1993).

Consequently a design with maximum information on the parameter subsystem $K^{\prime} \theta$ solves the problem;

$$
\begin{gathered}
\text { Maximize } \phi_{p}\left(\mathrm{C}_{\mathrm{k}}(\mathrm{M}(\tau))\right) \text { with } \tau \in \mathrm{T} \\
\text { Subject to } \mathrm{C}_{\mathrm{k}}(\mathrm{M}(\tau)) \in \mathrm{PD}(\mathrm{m})
\end{gathered}
$$

Suppose $\eta(\alpha)$ satisfies the side condition $\mathrm{C}_{\mathrm{k}}(\mathrm{M}(\tau)) \in \mathrm{PD}(\mathrm{m})$ and write $\mathrm{C}_{\mathrm{j}}=\mathrm{C}_{\mathrm{k}}\left(\mathrm{M}\left(\eta_{j}\right)\right)$ for $\mathrm{j}=(1,2,3)$. For all $\mathrm{p} \in(-$ $\infty ; 1], \eta(\alpha)$ solves problem (11) if and only if;

$$
\operatorname{trace}_{0} C_{j} C^{p-1} H_{0}^{\prime}\left\{\begin{array}{l}
=\operatorname{trace}_{0} C^{p} H_{0}^{\prime} \text { for all } j \in \partial(\alpha) \\
\leq \text { trace } H_{0} C^{p} H_{0}^{\prime} \quad \text { otherwise }
\end{array}\right.
$$


(T. K. , 2004).

\section{Construction of the design}

We consider the weighted centroid design $\eta(\alpha)=\sum_{j=1}^{3} \alpha_{j} \eta_{j}=\alpha_{1} \eta_{1}+\alpha_{2} \eta_{2}+\alpha_{3} \eta_{3}$ with three elementary centroids (captured from the support points):

$$
\eta_{1}=\left\{\left(\begin{array}{l}
1 \\
0 \\
0
\end{array}\right),\left(\begin{array}{l}
0 \\
1 \\
0
\end{array}\right), \quad\left(\begin{array}{l}
0 \\
0 \\
1
\end{array}\right)\right\}, \eta_{2}=\left\{\left(\begin{array}{l}
1 / 2 \\
1 / 2 \\
0
\end{array}\right),\left(\begin{array}{l}
1 / 2 \\
0 \\
1 / 2
\end{array}\right),\left(\begin{array}{l}
0 \\
1 / 2 \\
1 / 2
\end{array}\right)\right\} \text { and } \eta_{3}=\left\{\left(\begin{array}{l}
1 / 3 \\
1 / 3 \\
1 / 3
\end{array}\right)\right\} .
$$

These designs discovered by ( (Scheffe', 1958) and (H., 1963)), are exchangeable and invariant under permutations, (T. K. , 2002). Weighted centroid designs are exchangeable.

The moment matrices for $\eta_{1}$ and $\eta_{2}$ are:

$$
M\left(\eta_{1}\right)=\left(\begin{array}{ccccccccc}
1 / 3 & 0 & 0 & 0 & 0 & 0 & 0 & 0 & 0 \\
0 & 0 & 0 & 0 & 0 & 0 & 0 & 0 & 0 \\
0 & 0 & 0 & 0 & 0 & 0 & 0 & 0 & 0 \\
0 & 0 & 0 & 0 & 0 & 0 & 0 & 0 & 0 \\
0 & 0 & 0 & 0 & 1 / 3 & 0 & 0 & 0 & 0 \\
0 & 0 & 0 & 0 & 0 & 0 & 0 & 0 & 0 \\
0 & 0 & 0 & 0 & 0 & 0 & 0 & 0 & 0 \\
0 & 0 & 0 & 0 & 0 & 0 & 0 & 0 & 0 \\
0 & 0 & 0 & 0 & 0 & 0 & 0 & 0 & 1 / 3
\end{array}\right)
$$

and

$$
M\left(\eta_{2}\right)=\left(\begin{array}{ccccccccc}
1 / 24 & 1 / 48 & 1 / 48 & 1 / 48 & 1 / 48 & 0 & 1 / 48 & 0 & 1 / 48 \\
1 / 48 & 1 / 48 & 0 & 1 / 48 & 1 / 48 & 0 & 0 & 0 & 0 \\
1 / 48 & 0 & 1 / 48 & 0 & 0 & 0 & 1 / 48 & 0 & 1 / 48 \\
1 / 48 & 1 / 48 & 0 & 1 / 48 & 1 / 48 & 0 & 0 & 0 & 0 \\
1 / 48 & 1 / 48 & 0 & 1 / 48 & 1 / 24 & 1 / 48 & 0 & 1 / 48 & 1 / 48 \\
0 & 0 & 0 & 0 & 1 / 48 & 1 / 48 & 0 & 1 / 48 & 1 / 48 \\
1 / 48 & 0 & 1 / 48 & 0 & 0 & 0 & 1 / 48 & 0 & 1 / 48 \\
0 & 0 & 0 & 0 & 1 / 48 & 1 / 48 & 0 & 1 / 48 & 1 / 48 \\
1 / 48 & 0 & 1 / 48 & 0 & 1 / 48 & 1 / 48 & 1 / 48 & 1 / 48 & 1 / 24
\end{array}\right) .
$$

Defining matrix $\tilde{L}=\left(K^{\prime} K\right)^{-1} K^{\prime}$ where $\mathrm{K}$ is the earlier defined (equation 4) coefficient matrix,

$$
\tilde{L}=\left(K^{\prime} K\right)^{-1} K^{\prime}=\left(\begin{array}{ccccccccc}
1 & 0 & 0 & 0 & 0 & 0 & 0 & 0 & 0 \\
0 & 0 & 0 & 0 & 1 & 0 & 0 & 0 & 0 \\
0 & 0 & 0 & 0 & 0 & 0 & 0 & 0 & 1 \\
0 & 3 / 2 & 0 & 3 / 2 & 0 & 0 & 0 & 0 & 0 \\
0 & 0 & 3 / 2 & 0 & 0 & 0 & 3 / 2 & 0 & 0 \\
0 & 0 & 0 & 0 & 0 & 3 / 2 & 0 & 3 / 2 & 0
\end{array}\right)
$$

The information matrices for the designs $\eta_{1}$ and $\eta_{2}$ are obtained as follows: 


$$
C_{1}=C_{k}\left(M\left(\eta_{1}\right)\right)=\tilde{L}\left(M\left(\eta_{1}\right)\right) \tilde{L}^{\prime}=\left(\begin{array}{cccccc}
1 / 3 & 0 & 0 & 0 & 0 & 0 \\
0 & 1 / 3 & 0 & 0 & 0 & 0 \\
0 & 0 & 1 / 3 & 0 & 0 & 0 \\
0 & 0 & 0 & 0 & 0 & 0 \\
0 & 0 & 0 & 0 & 0 & 0 \\
0 & 0 & 0 & 0 & 0 & 0
\end{array}\right)
$$

and

$$
C_{2}=C_{k}\left(M\left(\eta_{2}\right)\right)=\tilde{L}\left(M\left(\eta_{2}\right)\right) \tilde{L}^{\prime}=\left(\begin{array}{cccccc}
1 / 24 & 1 / 48 & 1 / 48 & 1 / 16 & 1 / 16 & 0 \\
1 / 48 & 1 / 24 & 1 / 48 & 1 / 16 & 0 & 1 / 16 \\
1 / 48 & 1 / 48 & 1 / 24 & 0 & 1 / 16 & 1 / 16 \\
1 / 16 & 1 / 16 & 0 & 3 / 16 & 0 & 0 \\
1 / 16 & 0 & 1 / 16 & 0 & 3 / 16 & 0 \\
0 & 1 / 16 & 1 / 16 & 0 & 0 & 3 / 16
\end{array}\right)
$$

Using equations (13) and (14), we obtain the information matrix for the design $\eta(\alpha)$ from; $C_{k}(M(\eta(\alpha)))=\alpha_{1} C\left(M\left(\eta_{1}\right)\right)+\alpha_{2} C\left(M\left(\eta_{2}\right)\right)$, as

$$
C_{k}=C_{k}(M(\eta(\alpha)))=\left(\begin{array}{cccccc}
\frac{8 \alpha_{1}+\alpha_{2}}{24} & \frac{\alpha_{2}}{48} & \frac{\alpha_{2}}{48} & \frac{\alpha_{2}}{16} & \frac{\alpha_{2}}{16} & 0 \\
\frac{\alpha_{2}}{48} & \frac{8 \alpha_{1}+\alpha_{2}}{24} & \frac{\alpha_{2}}{48} & \frac{\alpha_{2}}{16} & 0 & \frac{\alpha_{2}}{16} \\
\frac{\alpha_{2}}{48} & \frac{\alpha_{2}}{48} & \frac{8 \alpha_{1}+\alpha_{2}}{24} & 0 & \frac{\alpha_{2}}{16} & \frac{\alpha_{2}}{16} \\
\frac{\alpha_{2}}{16} & \frac{\alpha_{2}}{16} & 0 & \frac{3 \alpha_{2}}{16} & 0 & 0 \\
\frac{\alpha_{2}}{16} & 0 & \frac{\alpha_{2}}{16} & 0 & \frac{3 \alpha_{2}}{16} & 0 \\
0 & \frac{\alpha_{2}}{16} & \frac{\alpha_{2}}{16} & 0 & 0 & \frac{3 \alpha_{2}}{16}
\end{array}\right)
$$

This matrix has a regular inverse,

$$
[C(M(\eta(\alpha)))]^{-1}=\left(\begin{array}{cccccc}
\frac{3}{\alpha_{1}} & 0 & 0 & \frac{-1}{\alpha_{1}} & \frac{-1}{\alpha_{1}} & 0 \\
0 & \frac{3}{\alpha_{1}} & 0 & \frac{-1}{\alpha_{1}} & 0 & \frac{-1}{\alpha_{1}} \\
0 & 0 & \frac{3}{\alpha_{1}} & 0 & \frac{-1}{\alpha_{1}} & \frac{-1}{\alpha_{1}} \\
\frac{-1}{\alpha_{1}} & \frac{-1}{\alpha_{1}} & 0 & \frac{2\left(8 \alpha_{1}+\alpha_{2}\right)}{3 \alpha_{1} \alpha_{2}} & \frac{1}{3 \alpha_{1}} & \frac{1}{3 \alpha_{1}} \\
\frac{-1}{\alpha_{1}} & 0 & \frac{-1}{\alpha_{1}} & \frac{1}{3 \alpha_{1}} & \frac{2\left(8 \alpha_{1}+\alpha_{2}\right)}{3 \alpha_{1} \alpha_{2}} & \frac{1}{3 \alpha_{1}} \\
0 & \frac{-1}{\alpha_{1}} & \frac{-1}{\alpha_{1}} & \frac{1}{3 \alpha_{1}} & \frac{1}{3 \alpha_{1}} & \frac{2\left(8 \alpha_{1}+\alpha_{2}\right)}{3 \alpha_{1} \alpha_{2}}
\end{array}\right)
$$

The slope matrix D as defined by equation (6) is obtained as

$$
D=\left(\begin{array}{ccccccccc}
2 t_{1} & t_{2} & t_{3} & t_{2} & 0 & 0 & t_{3} & 0 & 0 \\
0 & t_{1} & 0 & t_{1} & 2 t_{2} & t_{3} & 0 & t_{3} & 0 \\
0 & 0 & t_{1} & 0 & 0 & t_{2} & t_{1} & t_{2} & 2 t_{3}
\end{array}\right)
$$

A corresponding adjusted slope matrix $H_{0}=D K$ is thus given by; 


$$
H_{0}=\left(\begin{array}{cccccc}
2 t_{1} & 0 & 0 & \frac{2}{3} t_{2} & \frac{2}{3} t_{3} & 0 \\
0 & 2 t_{2} & 0 & \frac{2}{3} t_{1} & 0 & \frac{2}{3} t_{3} \\
0 & 0 & 2 t_{3} & 0 & \frac{2}{3} t_{1} & \frac{2}{3} t_{2}
\end{array}\right)
$$

To get the D-optimal design we employ the relation, that $\eta(\alpha)$ is $\phi_{p}-$ optimal for $K^{\prime} \theta$ in $\mathrm{T}$ if and only if;

$$
\text { trace } H_{0} C_{j} C^{p-1} H_{0}^{\prime}\left\{\begin{array}{l}
=\operatorname{traceH}_{0} C^{p} H_{0}^{\prime} \quad \text { for } j=1,2 \\
<\text { traceC }^{p} \text { otherwise }
\end{array}\right.
$$

From which, the unique D-optimal design for $K^{\prime} \theta$ is derived using the equation (putting $\mathrm{p}=0$ )

$$
\text { trace } H_{0} C_{j} C^{-1} H_{0}^{\prime}=\operatorname{trace}_{0} C^{0} H_{0}^{\prime}=\text { trace }_{0} H_{0}^{\prime} \quad \text { for } j=1,2
$$

The following results can be easily demonstrated using condition (16):

- $\quad$ For $\mathrm{j}=1$

$H_{0} C_{1} C^{-1} H_{0}^{\prime}=\frac{1}{3 \alpha_{1}}\left(\begin{array}{ccc}12 t_{1}^{2}-\frac{4}{3}\left(t_{1} t_{2}+t_{1} t_{3}\right) & -\frac{4}{3} t_{1}^{2} & -\frac{4}{3} t_{1}^{2} \\ -\frac{4}{3} t_{2}^{2} & 12 t_{2}^{2}-\frac{4}{3}\left(t_{1} t_{2}+t_{2} t_{3}\right) & -\frac{4}{3} t_{2}^{2} \\ -\frac{4}{3} t_{3}^{2} & -\frac{4}{3} t_{3}^{2} & 12 t_{3}^{2}-\frac{4}{3}\left(t_{1} t_{3}+t_{2} t_{3}\right)\end{array}\right)$, with

trace $H_{0} C_{1} C^{-1} H_{0}^{\prime}=\frac{1}{3 \alpha_{1}}\left[12\left(t_{1}^{2}+t_{2}^{2}+t_{3}^{2}\right)-\frac{8}{3}\left(t_{1} t_{2}+t_{1} t_{3}+t_{2} t_{3}\right)\right]=\frac{496}{27 \alpha_{1}}$ and

$H_{0} H_{0}^{\prime}=\left(\begin{array}{ccc}4 t_{1}^{2}+\frac{4}{9}\left(t_{2}^{2}+t_{3}^{2}\right) & \frac{4}{9} t_{1} t_{2} & \frac{4}{9} t_{1} t_{3} \\ \frac{4}{9} t_{1} t_{2} & 4 t_{2}^{2}+\frac{4}{9}\left(t_{1}^{2}+t_{3}^{2}\right) & \frac{4}{9} t_{2} t_{3} \\ \frac{4}{9} t_{1} t_{3} & \frac{4}{9} t_{2} t_{3} & 4 t_{3}^{2}+\frac{4}{9}\left(t_{1}^{2}+t_{2}^{2}\right)\end{array}\right)$, with

trace $_{0} H_{0}^{\prime}=\frac{44}{9}\left(t_{1}^{2}+t_{2}^{2}+t_{3}^{2}\right)=\frac{638}{27}$

The condition, trace $H_{0} C_{1} C^{-1} H_{0}^{\prime}=$ trace $_{0} H_{0}^{\prime}$ implies that $\frac{496}{27 \alpha_{1}}=\frac{638}{27}$, giving $\alpha_{1}=\frac{248}{319}$

- $\quad$ For $\mathrm{j}=2$;

$H_{0} C_{2} C^{-1} H_{0}^{\prime}=\frac{4}{9 \alpha_{2}}\left(\begin{array}{ccc}t_{2}^{2}+t_{3}^{2}+t_{1} t_{2}+t_{1} t_{3} & t_{1}^{2}+t_{1} t_{2} & t_{1}^{2}+t_{1} t_{3} \\ t_{2}^{2}+t_{1} t_{2} & t_{1}^{2}+t_{3}^{2}+t_{1} t_{2}+t_{2} t_{3} & t_{2}^{2}+t_{2} t_{3} \\ t_{3}^{2}+t_{1} t_{3} & t_{3}^{2}+t_{2} t_{3} & t_{2}^{2}+t_{2}^{2}+t_{1} t_{3}+t_{2} t_{3}\end{array}\right)$

with $\operatorname{trace~}_{0} C_{2} C^{-1} H_{0}^{\prime}=\frac{8}{9 \alpha_{2}}\left(t_{1}^{2}+t_{2}^{2}+t_{3}^{2}+t_{1} t_{2}+t_{1} t_{3}+t_{2} t_{3}\right)=\frac{142}{27 \alpha_{2}}$

The equation, $\operatorname{trace} \mathrm{H}_{0} \mathrm{C}_{2} \mathrm{C}^{-1} \mathrm{H}_{0}^{\prime}=\operatorname{trace}_{0} H_{0}^{\prime}$ implies that $\frac{142}{27 \alpha_{2}}=\frac{638}{27}$, giving $\alpha_{2}=\frac{71}{319}$

Therefore the unique D-optimal design for $K^{\prime} \theta$ is 


$$
\eta\left(\alpha^{(D)}\right)=\alpha_{1} \eta_{1}+\alpha_{2} \eta_{2}=\frac{248}{319} \eta_{1}+\frac{71}{319} \eta_{2} .
$$

The information matrix:

$$
H_{0} C H_{0}^{\prime}=\left(\begin{array}{ccc}
4 a t_{1}^{2}+4 b\left(t_{2}^{2}+t_{3}^{2}+2 t_{1} t_{2}+2 t_{1} t_{3}\right) & 4 b\left(t_{1}^{2}+t_{2}^{2}+2 t_{1} t_{2}\right) & 4 b\left(t_{1}^{2}+t_{3}^{2}+2 t_{1} t_{3}\right) \\
4 b\left(t_{1}^{2}+t_{2}^{2}+2 t_{1} t_{2}\right) & 4 a t_{2}^{2}+4 b\left(t_{1}^{2}+t_{3}^{2}+2 t_{1} t_{2}+2 t_{2} t_{3}\right) & 4 b\left(t_{2}^{2}+t_{3}^{2}+2 t_{2} t_{3}\right) \\
4 b\left(t_{1}^{2}+t_{3}^{2}+2 t_{1} t_{3}\right) & 4 b\left(t_{2}^{2}+t_{3}^{2}+2 t_{2} t_{3}\right) & 4 a t_{3}^{2}+4 b\left(t_{1}^{2}+t_{2}^{2}+2 t_{1} t_{3}+2 t_{2} t_{3}\right)
\end{array}\right)
$$

Where $a=\frac{8 \alpha_{1}+\alpha_{2}}{24}, b=\frac{\alpha_{2}}{48}, t_{1}^{2}=\frac{29}{18}$ and $t_{i} t_{j}=\frac{13}{36}$

The maximum of the D-criterion is $v\left(\phi_{0}\right)=\left(\frac{1}{5.964}\right)^{\frac{1}{3}}=0.5514$.

\section{Conclusion}

The design presented is highly efficient and can be employed as a design for a finite sample size. Of importance is to relate the weights to the number of support points for each centroid. However, the experimenter is cautioned to ensure high accuracy levels in the measurement of ingredient levels.

\section{References}

Draper, N. R., \& Pukelsheim, F. (1998). Mixture Models Based on Homogeneous Polynomials. Journal of Statistical Planning and Inference, 71, 303-311. https://doi.org/10.1016/S0378-3758(98)00012-3

Draper, N. R., Heilijers, B., \& Pukelsheim, F. (2000). Kiefer Orderinf of Simplex Designs for Mixture Models with Four Ingredients. Annals of Statistics, 28, 578-590. https://doi.org/10.1214/aos/1016218231

Henry, S. (1963). The Simplex-Centroid Design for Experiments with Mixtures. J. Roy. Statist. Soc. Ser., 25, $235-257$. https://doi.org/10.1111/j.2517-6161.1963.tb00506.x

Klein, T. (2004). Optimal Designs for Second-Degree Kronecker Model Mixture Experiments. Journal of Statistical Planing and Inference, 123, 117-131. https://doi.org/10.1016/S0378-3758(03)00145-9

Murty, V. N., \& Studden, W. J. (1972). Optimal Designs for Estimating the Slope of a Polynomial Regression. Journal of America Statistics Association, 67, 869-873. https://doi.org/10.1080/01621459.1972.10481308

Ott, L., \& Mendenhall, W. (1972). Designs for Estimating the Slope of a Second Order Linear Model. Technometrics, 14, 341-353. https://doi.org/10.1080/00401706.1972.10488920

Pukelsheim, F. (1993). Optimal Designs of Experiments. New York: wiley.

Scheffe, H. (1958). Experiments with Mixtures. J. Roy. Statist. Ser, $\quad$ B20, $344-360$. https://doi.org/10.1111/j.2517-6161.1958.tb00299.x

Sung, H. P., Hyang, S. J., \& Rabindra, D. (2009). Rotatability of Second Order Response Surface Regression Models with Corrected Error. Quality Technology \& Quantitative Management, 6(4), 471-492. https://doi.org/10.1080/16843703.2009.11673211

T., K. (2002). Invariant Symmetric Block Matices for Design of Mixture Experiments. Journal of Statistical Planning and Inference, 178-196.

T., K. (2004). Optimal Designs for Second-Degree Kronecker Model ixture Experiments. Journal of Statistical Planning and Inference, 123, 117-131. https://doi.org/10.1016/S0378-3758(03)00145-9

\section{Copyrights}

Copyright for this article is retained by the author(s), with first publication rights granted to the journal.

This is an open-access article distributed under the terms and conditions of the Creative Commons Attribution license (http://creativecommons.org/licenses/by/4.0/). 